\title{
Lessons from China: Understanding the Role of Mobile Applications in China's Response to COVID-19
}

\author{
Sophia Zhou, Isabelle Claude, Samuel Skinner, William Yang, Xianhan Jia
}

\begin{abstract}
The COVID-19 pandemic has impacted the society and human life profoundly. Since reported in China in December, 2019, the global confirmed cases have exceeded 24 million (as of August 27, 2020 according to Worldometers.info). The students from Mass Academy of Science and Math at WPI and Worcester Polytechnic Institute (WPI) worked with professors and students at Tsinghua University and Wuhan University of Technology to understand how mobile applications were used in China's response to COVID-19.
\end{abstract}

Many countries have launched COVID-19 apps with a wide range in how these apps work. While it is hopeful that these apps can assist in the fight against COVID-19, people are worried about user privacy. China implemented "health code" systems, which assigned neighborhoods and citizens a specific health code meant to indicate their risk of having been exposed to COVID-19. These health code systems were hosted on the popular apps WeChat and Alipay, each with billions of users. Some experts argued that China's use of mobile applications was essential in its successful combat against COVID-19.

In this study, a public survey and an expert interview guide were created after literature and background research. The survey and interview guide were revised multiple times with piloting at limited scale before implemented broadly. The collected data were analyzed using well established coding and numerical analysis methods. 
The interviews revealed four important themes: personal privacy, community involvement, government involvement, and situational specificity. It was found that balance between collecting and utilizing personal information, and protecting this information, was a key concern. In addition, close work between local communities and the national government was essential. Mobile applications assisted in communicating and coordinating, but did not replace the work of people such as delivery drivers and contact tracers. Our results also showed that there was room for improvement, especially accessibility to elderly or those unfamiliar with technology.

Similar results were obtained by our survey. It was slightly surprising to find that the apps were mostly used for "accessing information on COVID-19". In addition, respondents overwhelmingly identified "information" as the most valuable feature of COVID-19 apps. Both interview and survey results revealed the importance of providing information as a primary function of COVID-19 apps.

Based on our findings we distilled four main lessons: mobile applications should assist in existing COVID-19 responses, inform users, protect user's personal information, and adapt to their environment.

We recommend that public health officials and app developers take these lessons into consideration when developing COVID-19 related mobile applications. In addition, we encourage researchers to utilize our report as a jumping off point for further research. Acknowledgements: The authors would like to thank the professors at WPI, Tsinghua University and WUT for their guidance, student researchers at WUT for their invaluable assistance in translating and disseminating our survey, and all of the experts we interviewed for sharing their insights and experiences. 